\title{
La enseñanza de la lectura y la escritura integrada a la formación profesional
}

\author{
Marisol Perassi ${ }^{a}$, María Ester Cariello ${ }^{a}$; Liliana Castañeda ${ }^{a} \&$ Claudia Bonell ${ }^{b}$ \\ ${ }^{a}$ Cátedra de Comprensión Lectora y Producción Escrita, Grupo de Investigación y Desarrollo en Enseñanza de la Ingeniería, Facultad de Ingeniería, \\ Universidad Nacional de Entre Rios, Oro Verde,Entre Ríos, Argentina.mperassi@bioingenieria.edu.ar, cariellome@bioingenieria.edu.ar, \\ lcastaneda@bioingenieria.edu.ar \\ ${ }^{b}$ Cátedra de Comprensión Lectora y Producción Escrita, Facultad de Ingeniería, Universidad Nacional de Entre Rios, Oro Verde, Entre Ríos, Argentina. \\ cbonell@bioingenieria.edu.ar
}

\begin{abstract}
Resumen - El objetivo de este trabajo es describir las características y los resultados de la experiencia didáctica llevada cabo en la asignatura Comprensión Lectora y Producción Escrita, orientada a integrar la enseñanza en lectura y escritura a la formación profesional específica de las carreras en las cuales se inserta (Bioingeniería y Lic. en Bioinformática de la FI-UNER). Se presentan dos actividades, la producción grupal de un Trabajo monográfico y la observación de presentaciones de Proyectos Finales y Tesinas, así como los resultados de su implementación con alumnos en el año 2014. Se concluye que ambas actividades favorecieron tanto el logro de los objetivos específicos de lectura y escritura como el acercamiento de los estudiantes a temáticas propias de la profesión.
\end{abstract}

Palabras Clave-enseñanza universitaria,lectura; escritura académica, integración curricular.

Recibido: 15 de noviembre de 2015. Revisado: 27 de enero de 2016. Aceptado: 30 de agosto de 2016.

\section{The teaching of reading and writing integrated into professional training}

Abstract- This work aims to describe the characteristics and results of a didactic experience carried out in the subject Comprensión Lectora y Producción Escrita (Reading Comprehension and Writing), which integrates reading and writing into professional training related to courses included in the Bioengineering and Bioinformatics curricula at Faculty of Engineering UNER. Two activities are shown: a group production of a Monograph and the observation of the presentation of Final Projects and Dissertations. Furthermore, the results of this implementation with students during 2014 are shown. It is concluded that both activities helped the studentsachieving the specific objectives of reading and writingand become familiar with topics closely related to their profession.

Keywords- professional training, reading,academic writing, curriculum integration.

\section{Introducción}

La Bioingeniería y la Lic. en Bioinformática son carreras científico-tecnológicas que incluyen en sí mismas diversas disciplinas del campo de las ciencias exactas y naturales, como la matemática, la química, la informática, la biología, la electrónica, la física, la genética, etc. Según los Planes de estudio vigentes de ambas carreras en la Facultad de Ingeniería de la Universidad Nacional de Entre Ríos, la Bioingeniería pretende la formación de un profesional capaz de realizar el diseño y la producción de productos para la salud, diseñar, verificar y mantener las instalaciones hospitalarias, realizar, dirigir y participar en programas de investigación básica, clínica y aplicada vinculada al diseño y desarrollo de productos médicos en el área de la salud humana y animal, entre otras actividades. La Lic. en Bioinformática persigue la formación de un profesional de la informática aplicada a la biologíacapaz de integrar equipos de trabajo en modelización de estructuras y procesos de interés biológico y en diseños computacionales que permitan su estudio.

La asignatura Comprensión Lectora y Producción Escrita (CLyPE) integra el primer año de las carreras Bioingeniería y Lic. en Bioinformática de la Facultad de Ingeniería de la Universidad Nacional de Entre Ríos (FI-UNER), Argentina. Sus propósitos son ofrecer herramientas que favorezcan el proceso de aprendizaje de las demás asignaturas de la carrera y contribuir a que los estudiantes valoren la relevancia de la lectura y la escritura como prácticas necesarias para aprender, comprender y pensar, particularmente en el ámbito académico. Estos propósitos se orientan a la resolución de la situación problemática que se ha detectado en los alumnos ingresantes a nuestra facultad: dificultades en la lectura comprensiva de textos extensos y complejos y en la escritura como forma de expresión de sus conocimientos, constituyéndose estas dificultades en obstáculos para el aprendizaje.

Nuestro diagnóstico coincide con el de otros autores. Arnoux expresa: "La lectura es una de las prácticas de mayor presencia en la vida universitaria. Uno de los mayores obstáculos que debe sortear el alumno que inicia sus estudios superiores es precisamente adquirir el hábito de la lectura. De la lectura sostenida, profunda, crítica, analítica, de textos extensos, complejos, ante los que en la mayoría de los casos es la primera vez que se enfrenta" [1]. Para Carlino, la escritura

Como citar este artículo: Perassi, M., Cariello, M. E., Castañeda, L. \& Bonelld, C.. La enseñanza de la lectura y la escritura integrada a la formación profesional. Revista Educación en Ingeniería 12 (23) 4-8, Febrero, 2017. 
no es sólo un medio de comunicación, sino que supone análisis y transformación de lo que se sabe, de allísu potencialidad para favorecer los aprendizajes, en tanto al escribir se ponen en marcha procesos comola relación entre lo que ya sabemos con lo nuevo que queremos transmitir mediante la escritura,lo que no siempre ocurre en ausencia de producción escrita[2]. Rapetti y Velez sostienen que muchas veces en la universidad se observan prácticas de lectura y de escritura que no focalizan en la participación activa de los estudiantes respecto de la construcción y reconstrucción del sentido de texto, sino que la formación termina basándose en lecturas y escrituras de tipo mecánicas, priorizando la memorización y repetición del contenido de los textos por sobre la comprensión lectora y la potencialidad de la escritura para el aprendizaje [3].

La lectura, en tanto es un proceso interactivo entre texto y lector [4], toma características particulares de acuerdo al ámbito donde se desarrolla. Por esta razón, en la asignatura se ha propuesto articular la enseñanza de la lectura y la escritura con temas y espacios formativos propios de los planes de estudios de las carreras que han elegido nuestros estudiantes.

El aprendizaje significativo es opuesto al aprendizaje repetitivo, producto de la memorización mecánica[5], al igual sucede con la comprensión lectora en relación con la lectura repetitiva o mecánica. El aprendizaje significativo que aspiramos para nuestros estudiantes, está íntimamente ligado a la comprensión lectora, en tanto ambos suponen un sujeto que se apropia del contenido de lectura o estudio, atribuyéndole significados subjetivos a aquello que existe objetivamente. "Es imprescindible que el lector encuentre sentido en efectuar el esfuerzo cognitivo que supone leer" [4] y para ello es fundamental el contenido del texto.

Las actividades específicas que se han diseñado e instrumentado en CLyPE pretenden ser significativas y motivadoras para el alumno, en parte por estar relacionadas a lo que visualiza como parte de su futuro profesional. Se aspira a que poner en contacto a los ingresantes con temáticas propias del campo profesional de la carrera que cursan, contribuya de manera positiva a su motivación.

El objetivo de este trabajo es valorar el impacto en la formación de los estudiantes,de la aplicación de un diseño didáctico orientado a integrar al desarrollo de la lectura comprensiva y la producción escrita, temas específicos de las carreras en las que se dicta la asignatura.

\section{Metodología}

El diseño didáctico comprendió dos actividades que se suman a las específicas de metodologías de estudio.

\subsection{Producción grupal de un Trabajo monográfico}

El Trabajo monográfico consistió en la producción grupal de un texto en el que los estudiantes debieron desarrollar un tema relacionado con la carrera que están cursando, a su elección. Se realizó a lo largo del cursado, en cuatro etapas, para cada una de las cuales deben presentar un informe escrito. La versión final se presentó de manera escrita y también oralmente ante sus compañeros y docentes.
Para elaborar el trabajo, los alumnos, organizados en grupos de no más de 3 integrantes, debieron recuperar contenidos desarrollados en la asignatura, tales como: géneros académicos y sus estructuras, planificación textual, búsqueda y análisis de materiales bibliográficos, evaluación de la confiabilidad de las fuentes, revisión textual, elaboración de citas y referencias bibliográficas, elaboración de resúmenes, entre otros.

Para la evaluación de esta producción, se valoró fundamentalmente la pertinencia de las fuentes de información utilizadas, la claridad en la expresión escrita y oral, y la definición clara del discurso propio y ajeno.

En cada una de las etapas, los docentes realizaron devoluciones con comentarios, sugerencias, e indicaciones que permiten a los alumnos reorientar su producción y mejorarla etapa tras etapa. Como primer paso, se orientó a los estudiantes en la elección del tema, que dentro de los relacionados directamente con la carrera, respondía también a inquietudes o intereses del grupo. Se los asistió en la búsqueda bibliográfica, para lo cual consultan la biblioteca de la facultad y bases de datos online de revistas científico-académicas. Se promovió la revisión crítica de la información, en cuanto a su adecuación para ser utilizada en la monografía y confiabilidad, cuando no proviene de las fuentes mencionadas. Para la exposición oral, se los orientó también en el uso adecuado de recursos visuales o audiovisuales de apoyo a la presentación.

Para su valoración, se tuvo en cuenta el grado en que los estudiantes lograron:

Respetar la estructura y pautas de formato establecidas.

Utilizar ortografía y gramática correctas.

Redactar con orden y coherencia.

Comprender del tema.

Seleccionar fuentes de información confiables.

Integrar la información recabada de las distintas fuentes consultadas.

Adecuar el discurso a los destinatarios.

Distinguir claramente el discurso propio del ajeno.

Realizar las entregas en los momentos previstos.

\subsection{Observación de presentaciones de Proyectos Finales de Bioingeniería o Tesinas de Bioinformática}

Para la finalización de las carreras de Bioingeniería y Bioinformática de la FIUNER, los estudiantes tienen que cumplir el requisito de elaborar un Proyecto Final, en el primer caso, y una Tesina, en el segundo. Ambas son instancias curriculares obligatorias en los respectivos planes de estudio que finalizan con una presentación oral y pública en la institución ante un jurado evaluador, y a la cual asisten otros estudiantes, docentes, familiares y amigos de los autores.

Los estudiantes de la asignatura CLyPE, del primer año de ambas carreras, tuvieroncomo actividad obligatoria la observación de al menos una de estas presentaciones durante el año académico.

Los alumnos asistieron a las presentaciones acompañados por sus tutores pares (estudiantes avanzados de ambas carreras), para realizar una observación y para poder conversar con ellos sobre las percepciones, dudas e intereses relacionados con las carreras, que les surgen a partir de esas presentaciones. 
Luego, en grupos de hasta 4 integrantes, elaboraron un informe, respondiendo a tres preguntas básicas que conocidas con anticipación. Esto implicóreflexionar sobre lo observado y escuchado en la presentación. La primera pregunta, les requierió mencionar y describir a grandes rasgos el tema de la presentación. La segunda indagó sobre los conceptos y /o procedimientos que no pudieron comprender, y requiere que los identifiquen. La tercera les solicitó formular sus propias preguntas, surgidas de lo que ellos pudieron interpretar o reconocer luego de la presentación.

Para valorar esta actividad se tuvo en cuenta el grado en que lograron:

Describir el tema de la presentación.

Explicitar lo que no pudieron comprender.

Formular preguntas pertinentes.

Otra valoración de la experiencia didáctica lo constituye la percepción de los estudiantes sobre la misma. Se analizaron entonces las opiniones vertidas en la encuesta que anualmente realiza la institución a los estudiantes, sobre distintos aspectos de las asignaturas que han cursado.

\section{Resultados}

Se presentan aquí los resultados de la aplicación de las dos actividades mencionadas y de la encuestadurante el año académico 2014. En total 74 estudiantes cursaron la asignatura, correspondiendo 64 a Bioingeniería y 10 a Bioinformática.

Para la Producción del Trabajo monográfico, se conformaron 29 grupos. Como muestra la Tabla 1, el 45\% de los grupos, eligió temas relacionados al equipamiento biomédico. El 24\%, trabajó sobre prótesis y órtesis. El 14\%, los estudiantes de Bioinformática, temas de la Biología. El 17\% restante, trabajó sobre temas diversos del ámbito de la Bioingeniería.

En la primera etapa, la totalidad tuvo dificultades respecto de los materiales bibliográficos seleccionados. Estas fueron de dos tipos: fuentes que al ser analizadas desde los criterios de confiabilidad, no resultaban válidas, o bien, fueron confiables pero de una complejidad demasiado elevada para alumnos de primer año.

En cuanto a la selección de los temas, la mayor dificultad

estuvo en encontrar un punto adecuado entre lo general y lo particular.

En la segunda etapa, la mayor dificultad estuvo en la elaboración de un texto propio a partir de las fuentes consultadas sobre el tema elegido, distinguiendo con claridad el discurso ajeno y el discurso propio, incorporando las citas correspondientes.

Otra dificultad importante fue integrar la información recabada de las distintas fuentes consultadas en un nuevo texto, respetando los diferentes conceptos e ideas, pero en una redacción integrada.

En todos los casos fueron necesarias correcciones tanto en ortografía y gramática como en pautas de formato.

El 31\% de los grupos logró una producción que respetaba todas las pautas establecidas en la tercera entrega, obteniendo una calificación Muy Bueno o superior. De los restantes, el
Tabla 1.

Áreas temáticas elegidas por los alumnos para sus trabajos monográficos

\begin{tabular}{|c|c|c|c|c|c|}
\hline Área & $\begin{array}{c}\text { Equipamiento } \\
\text { médico }\end{array}$ & $\begin{array}{c}\text { Prótesis y } \\
\text { órtesis }\end{array}$ & Biología & Otros temas & Total \\
\hline Temas & $\begin{array}{c}\text { *equipos de } \\
\text { rayos X } \\
\text { * gammagrafía } \\
\text { salival } \\
\text { * jeringas sin } \\
\text { agujas } \\
\text { *máquina de } \\
\text { hemodiálisis } \\
\text { * tomógrafo } \\
\text { *catéter de } \\
\text { Swan-Ganz } \\
\text { *incubadoras } \\
\end{array}$ & $\begin{array}{l}\text { *implantes } \\
\text { cocleares } \\
\text { * implantes } \\
\text { traumat. } \\
\text { * prótesis de } \\
\text { rodilla } \\
\text { * mano } \\
\text { robótica } \\
\text { *corazón } \\
\text { artificial }\end{array}$ & $\begin{array}{l}\text { *células } \\
\text { madre } \\
* \\
\text { proteínas } \\
* \text { virus. }\end{array}$ & $\begin{array}{c}\text { *Biomecánica } \\
\text { aplicada al } \\
\text { deporte } \\
\text { * inteligencia } \\
\text { artificial } \\
\text { * medicina } \\
\text { nuclear } \\
\text { * residuos } \\
\text { hospitalarios }\end{array}$ & \\
\hline $\begin{array}{l}\mathrm{n}^{\circ} \mathrm{de} \\
\text { trab. }\end{array}$ & 13 & 7 & 4 & 5 & 29 \\
\hline$\%$ & $45 \%$ & $24 \%$ & $14 \%$ & $17 \%$ & $100 \%$ \\
\hline
\end{tabular}

Fuente: Los autores

$52 \%$, necesitó realizar correcciones y hacer correctamente una cuarta entrega del trabajo, logrando así la promoción de la asignatura. Los restantes 5 grupos no lograron realizar el trabajo monográfico en tiempo y forma, no alcanzando la promoción.

En las presentaciones orales de los trabajos, todos los grupos lograron exponer el tema haciendo uso de recursos visuales o audiovisuales. Repartieron la palabra entre los integrantes del grupo, acompañaron de manera coordinada sus explicaciones orales con las imágenes y respetaron el tiempo de exposición.

En el caso de la actividad de observación de Proyectos Finales y Tesinas, los resultados (Tabla 2) muestran que la totalidad de los informes realizados por los estudiantes evidenció un nivel de comprensión dentro de lo esperado. Pudieron describir básicamente el tema de la presentación y algunos aspectos sobresalientes de la misma.

Respecto a identificar cuestiones que no pudieron comprender, la mayoría de los grupos, el $73 \%$, identificó distintas cuestiones técnicas. El 27\% restante, manifestó haber comprendido la exposición en su totalidad, no obstante, al formular preguntas, se evidenció la falta de comprensión de puntos que abarcó la presentación.

Todos los grupos formularon preguntas pertinentes. En uno de los grupos fueron de un nivel más elemental que el esperado. Solo un grupo mostró sus dudas sobre aspectos metodológicos de la investigación llevada a cabo para la formulación del proyecto. Un tercio logró formular alguna pregunta respecto a la posibilidad de aplicación del diseño del proyecto, en una situación por ellos conocida que identificaron como problemática.

Tabla 2.

Logros de los estudiantes en el trabajo de observación de proyectos finales o tesinas

\begin{tabular}{cc}
\hline Preguntas & Porcentaje de logro \\
\hline $\begin{array}{c}\text { Identificar y describir básicamente el tema } \\
\text { de la presentación }\end{array}$ & 100 \\
$\begin{array}{c}\text { Identificar cuestiones que no pudieron } \\
\text { comprender }\end{array}$ & 73 \\
$\begin{array}{c}\text { Elaborar preguntas pertinentes a la } \\
\text { presentación }\end{array}$ & 100 \\
\hline Fuente: Los autores
\end{tabular}

Fuente: Los autores 
Tabla 3 .

Opiniones de los alumnos respecto de la articulación de la asignatura con la carrera

\begin{tabular}{ccc}
\hline Categorías & Cantidad de alumnos & Porcentaje \\
\hline Muy Buena & 9 & $17 \%$ \\
Buena & 30 & $58 \%$ \\
Regular & 11 & $21 \%$ \\
Mala & 0 & $0 \%$ \\
NS/NC & 2 & $4 \%$ \\
Total & 52 & $100 \%$ \\
\hline
\end{tabular}

Fuente: Los autores

La encuesta realizada por la institución, fue respondida por el $70 \%$ de los alumnos que cursaron la asignatura en el año 2014. Los resultados muestran que el $75 \%$ consideró que la articulación de la asignatura con la carrera fue Buena o Muy Buena (Tabla 3).

\section{Discusión}

Los temas elegidos por los estudiantes para producción del Trabajo monográfico, dan cuenta de los intereses y las representaciones que poseen sobre las carreras elegidas.

$\mathrm{Al}$ ser un trabajo realizado a lo largo del año y con varias instancias de devolución por parte de los docentes, se observó que todos los alumnos, en más o menos tiempo, lograron ir mejorando sus producciones progresivamente, hasta llegar a la versión final.

El tiempo extendido de la escritura permite que los estudiantes ejerciten en más de una oportunidad habilidades como la búsqueda de información (en las diferentes etapas del trabajo, seleccionando diferentes materiales), la planificación textual, la definición de objetivos de escritura o la revisión de lo escrito. Esto facilita ir superando paulatinamente la escritura lineal para ir adquiriendo otras características propias de escritores competentes, que no siempre utilizan un proceso de redacción lineal y ordenado, en el que primero se planifique la estructura del texto, después se escriba un borrador, luego se revise y se termine por hacer la versión final del escrito. Por el contrario, este proceso de escritura suele ser recursivo y cíclico [6].

Sobre la observación de Proyectos Finales y Tesinas, la identificación por parte de los alumnos de cuestiones que no comprenden, sumada a la pertinencia de las preguntas que formulan a partir de esta actividad y al "entusiasmo" que la misma les genera, permite reconocer la importancia de la vinculación temprana de los estudiantes que recién ingresan a la universidad con temas y actividades propias de las carreras que eligieron.

En efecto, ambas actividades contribuyen a dicha vinculación, tal como lo reconocen los mismos estudiantes en la encuesta realizada. Tanto la elaboración del Trabajo monográfico como la observación de proyectos y tesinas ofrece a los estudiantes nuevos elementos para continuar con una reflexión sobre la elección vocacional realizada, objeto de un nuevo trabajo.

\section{Conclusiones}

Tal como se ha mencionado ya, ambas actividades favorecieron el acercamiento de los estudiantes a temáticas propias de la profesión. Esto implica que tanto la observación de proyectos finales como la elaboración de la monografía abrieron preguntas en los estudiantes sobre las características específicas de la carrera, generaron procesos de búsqueda de información precisa, promovieron el acercamiento de los estudiantes que recién se inician al lenguaje propio de la profesión. Es sabido que una disciplina -así como una profesión- es un espacio discursivo y retórico, tanto como conceptual (Bogel y Hjortshoj citado en [2]), por lo cual la lectura y la escritura se constituyen en herramientas fundamentales para favorecer la formación en esa profesión.

Mediante estas actividades, la asignatura, además de aportar elementos para el aprendizaje de las estrategias básicas de lectura comprensiva y de producción de textos académicos, logra vincular a los estudiantes con temas y actividades propias de las carreras que eligieron y abrir nuevas instancias de reflexión sobre su elección vocacional.

Por todo lo dicho, la experiencia aquí analizada se presenta como un ejemplo de un posible modo de trabajo que potencia la integración de la enseñanza de la lectura y la escritura a la formación profesional, superando un modelo de formación universitaria basado en asignaturas aisladas como si se trataran de compartimentos estancos sin relación entre sí. La integración disciplinar y la significatividad social y subjetiva de los contenidos son dos aspectos imprescindibles para la formación de profesionales competentes en el contexto actual [7].

\section{Referencias}

[1] de Arnoux, E.N., Di Stefano, M. y Pereira, C., La lectura y la escritura en la universidad. Eudeba, 2004.

[2] Carlino, P., Escribir, leer y aprender en la universidad: Una introducción a la alfabetización. Buenos Aires, Argentina: Fondo de Cultura Económica, 2005.

[3] Rapetti, M. y Vélez, G., Leer para aprender y aprender a leer en la universidad. Entre lecturas estéticas y eferentes, Rev. Iberoam. Educ. Super., 3(7), pp. 113-128, 2012.

[4] Solé-Gallart. I., Estrategias de lectura, 13. Grao, 1992.

[5] Davini, M.C., Métodos de enseñanza. Buenos Aires, Argentina: Santillana, 2009.

[6] Cassany, D., Describir el escribir. Barcelona, España: Paidós, 1989.

[7] Mastache, A., Formar personas competentes. Buenos Aires, Argentina: Novedades Educativas, 2007.

M. Perassi, Obtuvo los títulos deProfesora y Licenciada en Ciencias de la Educación, en el año 2003, ambos en la Facultad de Ciencias de la Educación de la Universidad Nacional de Entre Ríos, Argentina. En la misma institución actualmente es maestranda en Docencia Universitaria, con tesis aprobada y en espera de su defensa oral. Se desempeña como asesora pedagógica desde el año 2004, y como docente desde el año 2006, en la Facultad de Ingeniería, Universidad Nacional de Entre Ríos. Integra el Grupo de Investigación y Desarrollo en Enseñanza de la Ingeniería de la Facultad de Ingeniería, Universidad Nacional de Entre Ríos, Argentina.

ORCID: 0000-0001-9683-8809

M.E. Cariello, obtuvo el título de Profesor en Ciencias Biológicas, expedido por la Facultad de Humanidades y Ciencias de la Educación de la Universidad Nacional de La Plata el 22 de julio de 1974. Docente de Biología en la Facultad de Ingeniería de la Universidad Nacional de Entre Ríos desde el año 1985 hasta 2002, como Jefe de Trabajos Prácticos. profesora adjunta de Histología y Anatomía desde julio de 2002 al 2005 y profesor asociado desde el 2005 hasta la fecha. Desarrolla tareas docentes en la cátedra Comprensión Lectora y Producción Escrita. En la Facultad de Ciencias Agropecuarias de la Universidad Nacional de Entre Ríos, Argentina cumple funciones como jefa de trabajos prácticos de Botánica Morfológica desde el año 1992 a la fecha. 
ORCID: 0000-0001-6493-4177

L. Castañeda, obtuvo el título de Bioquímica en 1986 y de MSc. en Didáctica de las Ciencias Experimentales en 2015, ambos en la Facultad de Bioquímica y Ciencias Biológicas de la Universidad Nacional del Litoral, Santa Fe, Argentina. Desde 1987 es docente de la Facultad de Ingeniería de la Universidad Nacional de Entre Ríos en asignaturas del área biológica y en Comprensión Lectora y Producción Escrita.

ORCID: 0000-0001-5447-0057

C.E. Bonell, recibió el título de Bioingeniera en 1993 y el título de MSc. en Ingeniería Biomédica en 2014, todos ellos de la Universidad Nacional de Entre

Ríos, Entre Ríos, Argentina. Entre 1993 y 2001 trabajó en la Administración Nacional de Alimentos y Tecnología Médica del Ministerio de Salud de Argentina. Desde su egreso en 1993 estuvo vinculada a la Facultad de Ingeniería de la Universidad Nacional de Entre Ríos primero como auxiliar docente de la cátedra de Electrotecnia de la carrera de Bioingeniería y siendo en la actualidad profesora adjunta en dicha cátedra. Realiza tareas de investigación en el Laboratorio de Ingeniería en Rehabilitación e Investigaciones Neuromusculares y Sensoriales en diversos proyectos. Colabora desde el año 2012 en la cátedra de Comprensión Lectora y Producción Escrita.

ORCID: 0000-0002-7025-9777 